
\title{
HYDRODYNAMIC STABILTTY OF MULTICOMPONENT DROPLET
GASIFICATION IN REDUCED GRAVITY
}

\author{
1. Aharon and B. D. Shaw \\ Mechanical and Aeronautical Engineering Deparment \\ University of Califomia \\ Davis, CA 95616
}

Paper 95-0145 presented at tho 33 rd AIAA Aerospace Sciences Mecting

\begin{abstract}
This investigation addresses the problem of hydrodynamic stability of a two-component droplet undergoing spherically-symmetrical gasification. The droplet components are assumed to have characteristic liquid species diffusion times that are large relative to characteristlc droplet surface regression times. The problem is formulated as a linear stability analysis, with a goal of predicting when spherically-symmetric droplet gasification can be expected to be hydrodynamically unstable from surfacetension gradicnts acting along the surface of a droplet which result from perturbations. It is found that for the conditions assumed in this paper (quasistoady gas phase, no intial droplet temperature gradients, diffusiondominated gasification), surface tension gradients do not play a role in the stability characteristics. In addition, all perturbations are predictod to decay such that droplets were hydrodynamically stable. Conditions are identified, however, that deserve more analysis as they may lead to hydrodynamic instabilities driven by capillary effects.
\end{abstract}

\section{INTRODUCTION}

Influences of volatility differences and liquid. phase transport on the vaporization and combustion behaviors of multicomponent droplets have been the subject of numerous investigations because of their potentlal importance to the efficlent use of fuel sprays as well as their fundamental interest. Many of these investigations have focused upon spherically cymmetric low Reynolds-number evaporation and combustion of multicomponent droplets. These investigations have demonstrated that liquidphase specles transport may be important when surface regression is fast compared to specles transport, and when significant volatility differences exist between llquid components. Landis and Mills [1], for example, report resulus of numerical analyses concerning spherjcally-symmetric evaporation of miscible binary droplets, demonstrating the effects of volatility differences and slow liquid-phase species transport. Law el al. have presented numerical and theorelical analyses for spherically-symmetric binary fuel evaporation with or without combustion [2-4]. Experimental work in this area has been active [see 5-12 for example]. Law el al. $[12,13]$ have also reported results regarding evaporation of methanol or other liquid droplets in humid air; a focus of these studies was the effects of water condensation on droplet evaporation. Reduced-gravity experiments on methanol droplet combustion where combustion-gencrated water was reasoned to have condensed onto the liquid droplet have been reported by Choi el al. [14].

Some of these investigations have demonstrated, for binary droplets with sufficlently different liquid component volatilities, that an initial transicnt period exists during which the more volatile component is preferentially cvaporated. During this time, as the droplet volume decreases, the fraction of the less volatile component in the droplet is increased relative 
to the initial state. Surface regression, when sufficiently rapid compared to liquid specics transport, causes a "concentration boundary layer" to dovelop near the droplet surface, where species profiles exhibit sharp spatial changes: tho loss volatile component is "swept up" In a thin layer because it cannot diffuse away from the droplet surface rapidly enough. It has been observed that under appropriate conditions (e.g., with spocies transport sufficiently slow compared to surfaco regression rates), a state may be attained where change? in droplet compositions occur slowly with respect to changes In droplet sizo $[1,2]$. This stale, which occur's after the initial transients have decayed, typically exhibits a concentration boundary-layer structure." For sufficiently small diffusion rates, this boundary-layer structure may persist for most of the remalning droplet lifetime.

Based on this observation, Law and Law [11] presented an asymptotic analysis concerning spherically-symmetric vaporization and combustion behaviors of multicomponent droplets for the case where liquid-phase species diffusion is slow compared to droplet surface regression rates. In thoir anglysis, Law and Law focused upon liquid-phaso behavior for the case where the boundary-layer structure changes slowly, following : decay of initial transients. A conclusion of this work is that once the boundary-layer composition profiles have been established in a droplet, the mass-flux fraction of ahy specles off the droplet surface is equal to tho Initial liquid-phase mass fraction of that specles prior to vaporization. The analysis of Law and Law [11] was extended by Shaw [15], who demonstrated that following decay of initial transients, the mass-flux fraction of any species off the droplet surface is instead approximately equal to the volume-averaged mass fraction of that specles in the droplet interior.

If temperature or species concentration gradlents exist at the surface of a liquid droplet (e.g., because of small perturbations in the environment or within the droplet), it is

"Here, "boundary layer" refers to the species concentration boundary layer near the droplet surface. possible for surface-tension gradients to cxist. If sufficiently intense, these surface. tension gradients can cause significant motions of the liquid-gas interface, influencing transport of species and energy in the gas and liquid phases. It is caplliary effects such as these that this paper is concerned with. Theoretical studies wlll be performed to determine the influence of capillary convection on droplet hydrodynamic stability, with a goal of determining how capillary convection may compromise the attainment of spherical symmetry. The goal of this research is to perform a linear stability analysis to determine whether tho sphericallysymmetrical state is stable with respoct to small perturbations in the flow variables. Viscous effects as well as the effects of surface tension gradients will be allowed. The analysis will consider only the portion of tho droplet history whore the sphericallysymmetrical spocies profiles may be considered quasl-steady. Earlier portions of the droplet history associated with transient liquid heating and the initial bulldup of the species concontration profiles will not be addressed.

It is noted that there have been many theoretical and experimental studies dealing with the motions of droplets and bubbles resulting from caplllary effects. A literature review has revealed, however, that most previous studies of droplet gasification have generally neglected effects of surface-tension gradients, though a few studies have appoared. Higuera and Linán [16] have presented a linear stability analysis of an unsupported and stationary droplet vaporizing in a hot stagnant atmosphere. Chai et al. [17] have also presented results pertaining to stability of evaporating droplets vaporizing in a stagnant environment. Lozinski and Matalon [18] presented analytical studies of spinning droplets with thermocapillary effects. Niazmand et al. [19] have recently presented numerical calculations for vaporizing droplets that are moving relative to their envirosiment. The calculations of Niazmand et al. showed that capillary flows can significantly affect droplet internal velocity and temperature llelds of vaporizing octane and methanol droplets, even when droplet initial Reynolds numbers 
(relative to the gas phase) are as large as 50. It is noted that following the initial publications of Nlazmand et al. [19], Shih and Megarldis [20] presented computational results on the effects of thermocapillary stresses and flows for nearly the same conditions as those considered by Niazmand et al. None of these previous studics have considered droplets with more than liquid species, which is the primary subject of this paper.

ESTIMATES OF THE IMPORTANCE OF CAPILLARY EFFECTS

Estimates of the importance of capillary effects may be made by considering a droplet of radius $R$. A temperature or spectes concentration gradlent along the liquid surface will produce a surface-tension gradient that will be balanced by surface shear stresses in the gas and liquid phases. For those estimates, gas-phase shear stresses will be assumed negligible relative to liquid shear stresses such that surfaco-tension gradients are primarily balanced by liquid shear. Neglect of gas-phase shear in surface force balanoes is justifled if the gas-phase viscosity is small relative to the liquid viscosity, a condition that is generally satisfied for subcritical conditions, and if liquld velocitics relative to the amblent gas are small, l.e., thero is not a thin momentum boundary layer in the gas phase. Liquidphase shear will be assumed to be charactorizod by $\mu_{L} u / R$, where $\mu_{L}$ is the liquid viscosity, u a velocity characteristic of the flow induced in the droplet and $u / R$ represents a characteristic velocity gradient al the droplet surface. Use of $R$ as a characteristic distance should be appropriate since for hydrocarbon and alcohol fuels of interest, the ratio of the cvaporation constant $\mathrm{k}$ (based on the droplet diameter, that is, $\mathrm{k}=$ $4 d\left(R^{2}\right) / d t$, where $t$ is time) to the liquid kinematic viscosity $V_{L}$ is of order unity or less (i.c., $\mathrm{k} / \mathrm{v}_{L} \leq 1$ ). Physically, if $\mathrm{k} / \mathrm{v}_{L} \leq 1$, momentum changes at the droplet surface should have sufficient time to influence the droplet interior before the droplet cvaporates. $A$ balance between liquid shear stress and sưrface tension gradients gives $u \sim \Delta \sigma / \mu_{\mathrm{L}} \sim$ $\tau / t_{c}$ where $\Delta \sigma$ is a characleristic difference in surface tension from one side of the droplet to the other, and $t_{c}$ is a characteristic time for a fluid particle to be convected hallway around the droplet periphery (surface-tension gradients aro characlerized by $\Delta \sigma / \mathbb{R}$, and gasphase shear is neglected assuming that the liquid-phase viscosity is much larger than the gas-phase viscosity). If we define $t_{b}=R^{2} / k$ as a characteristic gasification ilme of a droplet, we may expect that capillary effects aro negligible if $t_{b} / t_{c}=R \Delta \sigma /\left(k \mu_{L}\right) \ll 1$. Conversely, if $R \Delta \sigma /\left(k \mu_{L}\right) \geq 1$, caplllary effects may cause significant convective nows over the lifetime of a droplet.

The magnitude of $\Delta \sigma$ depends upon gradients of temperatures and species concentrations along the droplet surface. Let us flrst consider surface temperature gradients alone. For this case we may write $\Delta \sigma=|\partial \sigma / \partial \mathrm{T}|$ $\Delta T$, where $T$ is temperature, $\sigma$ surface tenslon, and $\Delta T$ is the temperature difference from one side of the droplet to the other. If this relation is used in the expression for to tha. we may solve for $\Delta \mathrm{T} \approx k \mu_{\mathrm{L}} /(\mathrm{R}|\partial \sigma / \partial \mathrm{T}|)$ as being required for $t_{b} / l_{c} \otimes 1$. For a $1 \mathrm{~mm}$ diameter hydrocarbon or alcohol droplet undergoing combustion, estimates suggest that $\Delta T=0.01 \mathrm{~K}$ is required to make $t_{b} / t_{c}=$ 1. These estimates are based upon the characteristic values $\mathrm{k}=10^{-6} \mathrm{~m}^{2} / \mathrm{s}, \mu_{\mathrm{L}}=4 \mathrm{x}$ $10^{-4} \mathrm{~kg} /(\mathrm{m} \mathrm{s})$, and $|\partial \sigma / \partial \mathrm{T}|=10^{.4} \mathrm{~N} /\left(\mathrm{m}^{\circ} \mathrm{K}\right)$. which are appropriate for near-atmospheric pressure conditions and droplet gasification in a hot environment or combustion. Since the ratio $t_{b} / l_{c}$ scales linearly with $\Delta T$, increases in $\Delta \mathrm{T}$ producc increases in $\mathrm{t}_{\mathrm{b}} / \mathrm{lc}_{\mathrm{c}}$. It thus appears that even very small temperature differences may induce significant convective flows.

We may also consider the effects of species concentration gradients along the droplet surface, neglecting temperature gradients. For simplicity, attention will be restricted to 
droplets composed of two miscible liquild components. For this case, we may write $\Delta \sigma$ $\mid \partial \sigma / \partial x_{1} \Delta x_{1}$ where $x_{1}$ is the mole fraction of component "one" at the llquid surface, and $\Delta x_{1}$ is the difference in $x_{1}$ from one side of the droplet to the other. Use of this result in the expression for $t_{b} / t_{c}$ yields $\Delta x_{1} \sim$ $k \mu_{\nu} / R$ b $\sigma / \partial x_{1} b$ for $t_{b} / l_{c}=1$.

Consider a methanol droplet of about $1 \mathrm{~mm}$ diameter. It is known that methanol (or other alcohol) droplets may absorb water from the gas phase. The characteristic values $\left|\partial o / \partial x_{1}\right|$ $=0.03 \mathrm{~N} / \mathrm{m}, \mathrm{k}=10^{.6} \mathrm{~m}^{2} / \mathrm{s}$, and $\mu_{\mathrm{L}}=4 \mathrm{x}$ $10^{-4} \mathrm{~kg} /(\mathrm{m} \mathrm{s})$ are appropriate for making order-of-magnitude estimates for methanolwater mixture droplets undergoing combustion at near atmospheric pressure. Uso of these values provides the result that $\Delta x_{l}=10^{-5}$ for $t_{b} / t_{c} \sim 1$. Hence, very small variations in water concentration along the surface of a methanol droplet may induce significant convective flows. Since the ratio $t_{b} / t_{c}$ scales linearly with $\Delta x_{1}$, larger values of $\Delta x_{1}$ produce increases in to/tc. Significant caplliary flows will negate the possibility of attaining liquid-phase spherical symmetry by enhancing mixing in an asymmetric and unknown manner. Estimates similar to those presented above for methanol-water mixtures have also been made for miscible binary hydrocarbon mixture droplets. These estimates suggest that for droplet components that vary signiflcantly in volatility, e.g., heptane-hexadecane, small differences in surface molo fractions of one component ( $\Delta x$ $\approx 10^{-4}$ ) may cause $t_{b} / t_{c}-1$. Such droplets (with significant volatility differentials) have been the subject of a number of reducedgravily experimental efforts which are striving for spherical symmetry.

Based upon the above estimates, it appears that small perturbations in temperature or species profiles along the surface of a binary miscible droplet may induce significant convectivo flows. If sufficiently intense. these convective flows will seriously compromise spherical symmctry, making interpretation of reduced-gravity experimental results (which are presumed to be spherically symmetrical) difficult. For these reasons, theoretical studies of the influences of capillary phenomena on droplet gasification and combustion are presented below. These studies will allow the influences of capillary phenomena to be delineated with greater precision, allowing for better intorpretation of reduced-gravity experimental results, and also for increased understanding of droplet rasiffication phenomena in gencral. For simplicity, the analyses are restricted to evaporation in a hot environment; effects of gas-phase combustion will bo considered af a later time. However, it is noted that the results presented here should be valid for combustion of droplets when the llame lies a significant distance away from the droplet surface, for example when droplets are burned in environments with low oxidizer concentrations.

In the following, the basic unperturbed solution for quasisteady droplet cvaporation in a hot onvironment will first be presented. This solution, which is presented for the convenience of the reader, is a summary of an analysis presented by Shaw [15]. After presontation of this solution, the viscous. flow governing equations will be perturbed and linearized. These equations will be solved and stability criteria will be developed.

\section{THB UNPERTURBED SOLUTION}

\section{Liquid-Phase Aralysis}

With the assumption of a constant liquid species diffusivity $D_{L}$ and also assuming that liquid densities do not vary with time, liquidphaso species conservation may be taken to be described by the partial differential equation and boundary conditions (where $y_{1}$ represents the mass fraction of species $\mathrm{i}$ )

$$
\frac{\partial y_{1}}{\partial r}=\frac{D_{l}}{r^{2}} \frac{\partial}{\partial r}\left(r^{2} \frac{\partial y_{1}}{\partial r}\right)
$$


$\left(D_{1} \frac{\partial y_{1}}{\partial r}+y_{1} \frac{d R}{d t}\right)_{r=R}=\delta_{1} \frac{d R}{d t},\left(\frac{\partial y_{1}}{\partial r}\right)_{r=0}=0$

and the initial condition $y_{1}(r, 0)=y_{10}$. Here, $y_{10}$ is taken for simplicity to be constant. The variable $\delta_{1}$ is the mass-flux fraction of species 1 from the droplet surface i.e.. $\delta_{1}=$ $(\mathrm{dm} / \mathrm{dt}) /(\mathrm{dm} / \mathrm{dt})$, where $\mathrm{m}$ is the droplet mass, $m_{1}$ the mass of component $I$ in the droplet, and $t$ the time. The droplet surface is regressing, so the location of the liquld-phase boundary is timo dependent. In the following, the droplet surface is fixed at unity with the trensformation $x=r / R$. Then with $\varepsilon=$ $D_{L} /[R(d R / d t)]$, liquid phase specles conservation is described by the Initial value problem

$\frac{\partial y_{1}}{\partial \tau}=\frac{e}{x^{2}} \frac{\partial}{\partial x}\left(x^{2} \frac{\partial y_{i}}{\partial x}\right)-x \frac{\partial y_{1}}{\partial x}$

$\left(\varepsilon \frac{\partial y_{1}}{\partial x}-y_{1}\right)_{x-1}=-\delta_{1},\left(\frac{\partial y_{1}}{\partial x}\right)_{x=0}=0$

$y_{1}(x, 0)=y_{10}$.

The physical time $t$ and the nondimensional

time $\tau$ are rolated by $t=\int_{0}^{t} d \tau /\left[d \ln \left(R_{0} / R\right) / d t\right]$. When d-square law surface regression holds. l.e., where the droplet surface area decreases linearly with time, $e=8 D_{l} / k$, where $k=$ $8 \mathrm{R}(\mathrm{dR} / \mathrm{d} \mathrm{t})$ is the evaporation constant. For this case it then follows that $U / t_{b}=1-e^{-2 \tau}$, where $t_{b}=4 R_{0}^{2} / k$ is the droplet evaporation Iffetime and $R_{0}$ is the initial droplet radius.

The situation of interest here is when liquidphase speoies transport is slow relative to droplet surface regression. Consider the second component to have a volatility that is low relative to the first component. The more volatile component will be initially preferentially vaporized, leaving the less volatile component bohind in the droplet. The droplet will become richer in the less volatile component relative to the initial state, with most of the lower-volatillty component left behind being collected within a boundary layer at the droplet surface. As surface regression procoeds, the surface mass fraction of the less volatile component builds. and a state wlll eventually be attained where vaporization of the less volatile component becomes slgnificant. Since both species will then vaporize vigorously, and liquid-phase diffusion is slow, further changes in droplet species profiles will occur slowly. Orily that part of the combustion history where both components are vaporizing vigorously with slowly-changing species profiles will be analyzed here. Analyses of transient boundary layer buildup have been given elsewhere [2I].

Inspection of $\mathrm{Eq}$. (2) suggests that once boundary layer growth has slowed considerably and surface conditions change slowly, further changes in species profiles over the droplet radius will occur over times $\Delta \tau$ which may be of order (1/e). The time period $\Delta \tau \sim O(1 / \varepsilon)$ may comprise the major portion of the remaining droplet lifetime in physical timo, since $E<<1$ is expected, as argued below. Because characteristic changes in $y$ aro of order unity or less, the magnitude of the partial derivative $\partial y / \partial \tau$ for these times may be estimated as $\left(\partial y_{l} / \partial \tau\right) S O(\varepsilon)$. Hence, it may be assumed that the boundary-layer structure which has evolved once vigorous vaporization of both components begins will be adequate for evaluating $\delta_{1}$. These observations are qualitatively supported by available numerical results [2], where the boundary-layer structures were observed to persist for most of the droplet lifetime, until near the end, when the more volatile component was vaporized much faster than the less volatile component.

Analysis of the boundary layer struclure proceeds by considering Eq. (2). Because $D_{L}$ is generally small compared to characteristic magnitudes of $R(d R / d t)$, it may be considered that $\varepsilon<1$. The "stretched" coordinate $z=(1-$ $x) / \varepsilon$ may then be inserted into Eq. (2). By using techniques of matched asymptotic 
expansions, it may be shown [15] that the mass-flux fraction $\delta_{i}$ may be expressed as

$\delta_{1}=y_{10}+3 e\left(y_{1-}-y_{10}\right)+O\left(e^{2}\right)$

while the asymptotic boundary-layer structure is provided by

$$
\begin{gathered}
y_{1}=y_{10}+ \\
\left(y_{1}-y_{10}\right) \exp (-z)\left[1+e\left(3 z+z^{2} / 2\right)+\ldots\right] .
\end{gathered}
$$

To complete the formulation of the model, the gas phase and the gashiguld coupling must be analyzed, as presented below.

\section{Gas Phase Analysis}

Por analysis of the gas phase, the simplifications of unity Lewis number, quasisteadiness i.e., neglect of the transient terms in the governing equations), negliglble body. forcos, negligible radiant heat flux, equal and constant spectfic heats and binary diffusion coefficients, and that diffusion oboys Fick's law will be employed. In the following, the subscript + denotes that conditions are evaluated on the gas side of the gas-liquild interface.

Assuming spherical symmetry, the energy and species conservation equations for the gas phase can respectively be written as

$$
\begin{aligned}
& \rho v \frac{\mathcal{C P}_{\mathrm{P}}}{\lambda} \frac{\mathrm{dT}}{\mathrm{dr}}=\frac{1}{\mathrm{r}^{2}} \frac{\mathrm{d}}{\mathrm{dr}}\left(\mathrm{r}^{2} \frac{\mathrm{dT}}{\mathrm{dr}}\right) \\
& v \frac{\mathrm{dY_{1 }}}{\mathrm{dr}}=\frac{\mathrm{D}_{\mathrm{B}}}{\mathrm{r}^{2}} \frac{\mathrm{d}}{\mathrm{dr}}\left(\mathrm{r}^{2} \frac{d \mathrm{Y}_{1}}{\mathrm{dr}}\right)
\end{aligned}
$$

where $p$ is density, $c_{p}$ is specific heat, $\lambda$ is thermal conductivity (assumed constant), $T$ is temperature, $r$ is the radial distance outward from the droplet center, $D_{8}$ is the gas-phase species diffusivity (assumed constant and equal for all specles), $Y_{1}$ the mass fraction of species $i$, and $v$ is the gas velocity. These equations are to integrated subject to the conditions that $T=T_{+}$and $Y_{1}=Y_{1+}$ at the droplet surface $(r=R)$ as well as $T=T_{\text {. }}$ and $Y_{1}=Y_{1 \infty}$ at infinity. The solutions to these equations car be written as

$\frac{T-T_{\infty}}{T_{+}-T_{\infty}}=\frac{Y_{1}-Y_{1-\infty}}{Y_{1+}-Y_{1 \infty}}=\frac{1-\exp (-\phi / r)}{1-\exp (-\phi / R)}$

where the assumption of unity Lewis number has been used and $\phi=r^{2} \rho v\left(c_{\rho} / \lambda\right)$. By applying the conditions for conservation of energy and species at the droplet surface $\left(\rho+v_{+} L=-\lambda(d T / d r)_{+}\right.$and $\delta_{i}=Y_{1+}$. $\left.\left(D_{\beta} / v\right)\left(d Y_{\gamma} / d r\right)_{+}\right)$it may be shown that $\phi=$ $\mathrm{R} \ln \left(1+\mathrm{B}_{\mathrm{T}}\right)=\mathrm{R} \ln \left(1+\mathrm{B}_{\mathrm{l}}\right)$ where $\mathrm{B}_{\mathrm{T}}=$ $c_{p}\left(T_{\infty}-T_{+}\right) / L$ and $B_{1}=\left(Y_{1+}-Y_{1 \infty}\right) /\left(\delta_{1}-Y_{1+}\right)$ are transfer numbers, $\delta_{l}$ is the mass-flux fraction of species $i$ in the gas phase, and $L=$ $\Sigma \delta_{1} L_{1}$ is the heat of vaporization of the liquid mixture at the droplet surface. It is noted that $B_{T}$ and $B_{1}$ are required to have the same numerical values.

From conservation of mass principles, it can also be shown that $k=8 \lambda \ln (1+B) /\left(\rho_{L} c_{p}\right)$ where $\mathrm{B}=\mathrm{B}_{\mathrm{T}}=\mathrm{B}_{1}$. It may be shown that the surface mass fraction of a droplet component Is calculated using $Y_{1+}=\left(B \delta_{i}+Y_{i-\infty}\right) /(1+B)$, while $\varepsilon$ is related to $B$ by

$\varepsilon=\left(D_{L} \rho_{L} c_{p}\right) /[\lambda \ln (1+B)]$.

\section{Gas/Liquid Coupling Analysis}

To complete the formulation, the coupling between the gas and liquid phases must be considered. For simplicity, in this analysis phase equilibrium and ideal solution behavior will be assumed to describe vaporization. Mole fractions (X) and mass fractions are related by the identity $\mathrm{X}_{1}=\left(\mathrm{Y}_{\mathrm{N}} / \mathrm{W}_{1}\right) /\left(\Sigma \mathrm{Y}_{\mathrm{j}} / \mathrm{W}_{\mathrm{j}}\right)$. Gas and liquid mole fractions will be related using Raoult's law and the Clausius. Clapeyron relation with constant vaporization enthalpy, giving 
$\mathrm{X}_{1-}=\mathrm{X}_{1+} \exp \left[\mathrm{L}_{1}\left(1 / \mathrm{T}_{+}-1 / \mathrm{T}_{\mathrm{b}_{\mathrm{i}}}\right) / \mathrm{R}_{l}\right]$

In $\mathrm{Eq} .(7), \mathrm{T}_{b_{1}}$ is the boiling temperature of component 1 at the amblent pressure, and $R_{1}$ is the gas oonstant for specles 1 . The phase equilibrium relations used here are not essential for the development of this model. Nonideal mixtures may be modeled, for example, with the use of activity cocfficients.

To solve for the spherically-symmetrical - evaporation state, we first specify y/o. C...2e this is done, values for the surface composition in the gas phase are assumed (subject to $\Sigma \dot{Y}_{1+}=1$ ). The surface tomperature is then terated until $\Sigma \delta_{1}=1$ is attalned for the gas phase. The gas-liquid coupling rolations are then used to calculate the surface composition on the liquid side of the gas-liquld interface. The parameter $e$ is then calculated using $\mathrm{Eq}$. (6), and a value for $y_{10}$ based upon the values found above is finally calculated from $\mathrm{Eq}$. (3). If this value is not sufficlently closs to the initially-specified value of $y_{10}$, the above procedure is repeated with new values for the surface composition in the gas phase. Calculations for various cases are presented by Shaw [15], where it is shown that the $O(e)$ correction to $\delta_{1}$ present in Eq.(3) is dominant when y/o is sufficiently small.

The spherically-symmetrical model has now been completely formulated. The results presented above are required for the perturbation analysis developed in the next section.

\section{LINEARIZED STABILITY EQUATIONS}

\section{Liquild Phase}

The conservation equations for the liquid are assumed to be the incompressible constantproperly relations appropriate for viscous flulds with negligible viscous dissipation. These equations are listed below.

$$
\begin{gathered}
\nabla \cdot \vec{v}=0 \quad \text { (Continuity) ' } \\
\frac{d \vec{v}}{d t}=-\nabla p+\mu \Delta \vec{v} \quad \text { (Momentum) } \\
\frac{d T}{d t}=\alpha_{j} \Delta \mathrm{T} \quad \text { (Energy) } \\
\frac{d y_{1}}{d t}=D_{1} \Delta y_{1} \quad \text { (Species) }
\end{gathered}
$$

It is to be noted that $\nabla$ represents the grudient operator while $\Delta$ represents the Laplacian operator. In addition, $\vec{v}$ is the volocity vector, $p$ is pressure, $T$ is tomperature, and $\alpha_{1}$ is the thermal diffusivity of the liquid. In addition, $\frac{d}{d t}=\frac{\partial}{\partial t}+\vec{v} \cdot \nabla$ is the Lagranglan derivative. Since the droplet surface is regressing, it is convenient (as outlined above) to define the normalized radial coordinate $x=r / R$. When this is done, the Lagrangian derivative is transformed to the form

$$
\frac{d}{d t}=\frac{\partial}{\partial t}+\frac{\vec{y}}{R} \cdot \nabla^{*}-x \frac{1}{R} \frac{d R}{d t} \frac{\partial}{\partial x}
$$

where $\nabla^{*}$ is the dimensionless gradient opcrator expressed in terms of $x$.

We will now perturb the liquid-phase variables by assuming that $\vec{v}=\vec{v}_{0}+\vec{v}^{\prime}$, $\mathrm{p}=\mathrm{p}_{0}+\mathrm{p}^{\prime}, \quad \mathrm{T}=\mathrm{T}_{\mathrm{o}}+\mathrm{T}^{\prime}, \quad \mathrm{y}_{1}=\mathrm{y}_{10}+\mathrm{y}_{1}^{\prime}$ where the subscript "o" denotes the solution for the spherically-symmetrical state and the superscript "prime" denotes the perturbation quantity. It is assumed that $\vec{v}_{0}=0, p_{0}=$ constant, $T_{0}=$ constant, and that $y_{10}$ is predicted by $\mathrm{Eq}$. (4). The assumption of constant $T_{0}$ is consisicnt with the Idea that carly transients associated with initial droplet heal-up have decayed.

Substitution of the perturbed variables presented above into the liquid-phase governing equations produces ovolution equations for the perturbation variables (note that "primes" will be omitted for brovity). In 
deriving these equations, the dimensionlcss variables $\vec{V}_{L}=(8 R / k) \vec{v}^{\prime}, P_{L}=8 p^{\prime} R^{2} /\left(k \mu_{L}\right)$ and $T_{L}=T^{\prime} / T_{. .}$are Introduced, where $T_{.}$is the amblent temperature and the subscripl " $\mathrm{L}$ " denotes liquid. The perturbation equations are linearized by noglecting products of the perturbation quanticies or their derivatives, and terms involving the spherically. symmetrical base solutions cancel. In addition, the dimensionless time $\tau$ defined earlier is introduced, though $\tau$ is rescaled as described below (note that $\exp (-\tau)=R / R_{0}$ ). The timescale for liquid-phase species diffusion is generally much greater than that for diffusion of energy. A characteristic time $\tau_{0}$ for species diffusion across the thickness $e$ of the boundary layer is simply $\tau_{c}=\varepsilon$ (on the $\tau$ timescalo). For analysis of the equations, we therefore introduce the rescaled time $\tilde{\tau}=\tau / \varepsilon$ to produce the final form of the equations, which are shown below.

$$
\begin{aligned}
& \nabla^{*} \cdot \overrightarrow{\mathrm{v}}_{\mathrm{L}}=0 \quad \text { (Continuity) } \\
& \Delta^{*} \overrightarrow{\mathrm{v}}_{\mathrm{L}} \cdot \nabla^{*} \mathrm{P}_{\mathrm{L}}=0 \quad \text { (Momentum) } \\
& \frac{1}{\varepsilon} \frac{\partial \mathrm{T}_{\mathrm{L}}}{\partial \tilde{\tau}}+x \frac{\partial \mathrm{T}_{\mathrm{L}}}{\partial \mathrm{x}}=\varepsilon \mathrm{L} \Delta^{*} \mathrm{~T}_{\mathrm{L}} \quad \text { (Energy) } \\
& \frac{1}{\varepsilon} \frac{\partial \mathrm{y}_{1}}{\partial \tilde{\tau}}+x \frac{\partial y_{1}}{\partial x}+\vec{V} \cdot \nabla^{*} \mathrm{y}_{10} \\
& =\varepsilon \Delta^{*} \mathrm{y}_{1} \text { (Species) }
\end{aligned}
$$

In dertving Eqs. (8) - (11), it has been assumed that the velocity field in the liguid phase will be a "creeping" flow (i.e. dominated by viscosity) such that fluid acceleration can be neglected in the first approximation. The variable le is the liquidphase Lewis number; Lo is assumed to be large relative to unity and the product ele is treated as $\mathrm{O}(1)$.

Solutions to Eqs. (8) - (11) will be sought assuming that cach scalar perturbation quantity $(Z(x, \theta, \phi))$ can be expressed in terms of a normal mode decomposition based upon expansions in terms of spherical harmonics

$Z=\sum_{n=0}^{\infty} \sum_{m=-n}^{n} Z_{n}(x) Y_{n}^{m}(\theta, \phi) e^{\beta_{n} \tilde{\tau}}$

whero $Z_{n}$ depends only on the transformed radial coordinate $x$ and $Y_{n}^{m}(\theta, \phi)$ is the spherical harmonic function. The function $Z_{n}$ represents a spatial amplitude of the perturbation mode $n$. A normal-mode analysis is appropriate herc since the unperturbed solution is quasisteady in the time variable $\tau$. A final goal of the analysis will be be to calculate the growth factor $\beta_{n}$, which will determine whether a perturbation will grow, decay, or remain sleady.

If we multiply the radial component of the momentum equation $\left(V_{x}\right)$ by $x$ and then iwice take the curl, we obtain the equation

$\left(\frac{d^{2}}{d x^{2}}+\frac{2}{x} \frac{d}{d x} \cdot \frac{n(n+1)}{x^{2}}\right)^{2}\left(x V_{L x}\right)=0$

where $V_{L x}$ is the amplitude of the amplitude of the velocity perturbation in the $x$-direction for the $n^{\text {th }}$ mode (for brevity the subscript " $n$ " is dropped from all equations involving amplitudes). In deriving this equation, use was made of the identity

$$
\begin{gathered}
\Delta\left[f(x) Y_{n}^{m}(\theta, \phi)\right]= \\
Y_{n}^{m}(\theta, \phi)\left[\frac{d^{2} f}{d x^{2}}+\frac{2}{x} \frac{d f}{d x} \cdot \frac{n(n+1)}{x^{2}} f\right]
\end{gathered}
$$

which is applicable for spherical harmonics and where $f(x)$ is an arbitrary function of $x$. Equation (13) is to be solved subject to the condition that the solution is finite at $x=0$. Integration yields

$V_{L x}=a_{1} x^{n-1}+a_{2} x^{n+1}$

where $a_{1}$ and $a_{2}$ are integration constants that will be determined by considering the solutions at the gas-liquid interface $(x=1)$. Interface conditions will be discussed later. 
The solution to the energy equation (Eq. (10)) may be developed by noting that we may reasonably expect that $e \ll 1$. To maintain a balance between dominant terms in Eq. (10), It can be assumed that time derivatives are at most $O(1 / \mathrm{L})$ ) In magnitude, which implies that the perturbed temperature fiold may be considered quasisteady in the first approximation. Wo will thus drop the transient tetm from $\mathrm{Eq}$. (10), allowing us to focus upon the effects of species perturbations on stability. Effects of temperature perturbations were studied by Higuera and Linán [16], who found that instabilities from temperature perturbations were important only when the base solution exhibits significant temperature gradients at tho droplet surface; spatial temperature gradionts are assumed to have decayed to negligible levels for the conditions under study here. If we now assume a solution of the form of $\mathrm{Eq}$. (12), the resulting ordinary differential equation for the amplitude $T_{L X}$ is

$$
\begin{gathered}
\frac{d^{2} T_{L x}}{d x^{2}}+\left(\frac{2}{x}-\frac{x}{E L e}\right) \frac{d T_{L x}}{d x} \\
-\frac{n(n+1)}{x^{2}} T_{L x}=0
\end{gathered}
$$

The point $x=0$ of Eq. (15) is a regular singular point, and it is possible to develop a Frobenius series solution about $x=0$. When this is done, it is found that the infinite-series solution actually converges very rapidly, and as a result the truncated expansion

$\left.T_{L x}=a\right\}\left\{x^{n}+\frac{1}{8 \operatorname{Le}} \frac{1}{16(2 n+3)} x^{n+2}\right\}$

may bo used with little loss in accuracy (as is a constant of integration). Applicability of Eq. (16) is based upon the condition that eLe is not small relative to unity; this condition is typically well-satisfied for droplet vaporization problems. Though not shown here, numerical solutions of Eq. (15) compare very well with Eq. (16) for the assumed conditions. In developing Bg. (16), the condition $T_{L X}=0$ was applied at the droplet center.
Substituting Eq. (12) into Eq. (11) yiclds the evolution equation for the amplitude $y_{i l}$ of the liquid-phase species perturbation

$$
\begin{gathered}
\beta_{n} y_{I L x}+\varepsilon \times \frac{d y_{11-x}}{d x} \\
+\left(a_{1} x^{n-1}+a_{2} x^{n+1}\right)\left(y_{1-}-y_{10}\right) \theta^{(x-1) / \varepsilon} \\
=\varepsilon^{2}\left(\frac{d^{2} y_{1 L x}}{d x^{2}}+\frac{2}{x} \frac{d y_{1 L x}}{d x}-\frac{n(n+1)}{x^{2}} y_{1 L x}\right) .
\end{gathered}
$$

In developing th:-equation, the leading-order solution for droplet species profiles was used (sec Eq. (4)). An asymptotic solution to Eq. (17) will be sought assuming that $\varepsilon \ll 1$, which is generally met for droplet gasification in hot environments. Examination of Bg. (19) indicates that a boundary-layer structure may be expected. The boundary layer will exist close to the droplet surface, and it is found that an appropriate scaling to allow analysis of the boundary-layer is provided by the transformation $x=1-\varepsilon z$, where $z \leq O(1)$ in the boundary layer. Using this transformation and retaining only the largest terms provides the leading-order inner problem

$$
\begin{aligned}
& \frac{d^{2} y_{1 L x}}{d z^{2}}+\frac{d y_{1 L x}}{d z}-\beta_{n} y_{1 L x}= \\
& \left(a_{1}+a_{2}\right)\left(y_{1-}-y_{10}\right) \exp (\cdot z) .
\end{aligned}
$$

The solution to Eq. (18) may be written as $y_{I L x}=a_{41} \exp \left(s_{4}(1-x) / \varepsilon\right)+a_{5 j} \exp \left(s_{5}(1\right.$. $x) / \varepsilon)-\left(a_{1}+a_{2}\right)\left(y_{1}-y_{10}\right) \exp [(x-1) / \varepsilon] / \beta_{n}$ where $a_{41}$ and $a_{51}$ are constants and $s_{4}=[-1$ $\left.\left(1+4 \beta_{n}\right)^{1 / 2}\right] / 2$ and $s_{5}=[-1+(1+$ $\left.\left.4 \beta_{n}\right)^{1 / 2}\right] / 2$. The leading-order outer solution to $\mathrm{Eg}$. (17) is simply $y_{1 \mathrm{Lx}}=0$. To match the inner and outer solutions we set ast $=0$, because for $\operatorname{Re}\left(\beta_{n}\right)>0$ the inner solution would grow exponentially fast for increasing z. The solution to Eq. (20) may therefore be expressed as

$$
\begin{aligned}
& y_{I L x}=a_{41} \exp \left[s_{4}(1-x) / \varepsilon\right]- \\
& \left(a_{1}+a_{2}\right)\left(y_{1-}-y_{10}\right) \exp [(x-1) / \varepsilon] / \beta_{n} .
\end{aligned}
$$


Gas Phase

Fur analysis of the gas phase, we will introduce the simplifylng assumptions that density changes, which are a result of temperature changes, may be neglected in the perturbation equations. We will also assume that tho vaporization is slow such that $\phi / R=$ $\ln (1+B) \ll 1$ (but where $\varepsilon=8 D, / k$ is also small relative to unity). This allows simplified analytical solutions to be found for the gas-phase perturbation variables. For analysis of the case where $\phi / R \geq 1$ and where density perturbations cannot be neglected, numerical inlegration of the perturbation equations is needed; this analysis is now in progress and will be presented elsewhere.

When $\phi / R \ll 1$, it is easily shown that a Reynolds number based upon the gas velocity and gas properties at the droplet surface is small relative to unity. Under these conditions, we may neglect the convective acceleration terms in the momentum equation. Wo will perturb the spherically-symmetrical solution by assuming that $\vec{v}=\vec{v}_{0}+\vec{v}^{\prime}$, $p=p_{0}+p^{\prime}, T=T_{0}+T^{\prime}$, and $Y_{1}=Y_{10}+Y_{1}^{\prime}$ where the subscript " $O$ " denotes the solution for the spherically-symmetrical state and the superscript "prime" denotes the perturbation quantily. If we further let $x=r / R$ and define the dimensionless perturbed variables $\vec{V}_{O}=\left(\rho_{d} / \rho_{L}\right)(8 R / k) \vec{V}^{\prime}, T_{O}=T / T_{0,}, Y_{10}=$ $Y_{i}$ and $P_{L}=8 p^{\prime} R^{2}\left(\rho_{G} / \rho_{L}\right) /\left(k \mu_{L}\right)$, the linearlzed conservation equations can be written as

$$
\begin{aligned}
& \nabla^{*} \cdot \overrightarrow{\mathrm{V}}_{\mathrm{O}}=0 \quad \text { (Continuity) } \\
& \Delta^{*} \overrightarrow{\mathrm{V}}_{\mathrm{O}}-\nabla^{*} \mathrm{P}_{\mathrm{O}}=0 \quad \text { (Momentum) } \\
& \Delta^{*} \mathrm{~T}_{\mathrm{O}}=\mathrm{O}(\phi / \mathrm{R}) \text { (Energy) }
\end{aligned}
$$

$$
\Delta Y_{10}=\alpha(\phi / R) \text { (Species) }
$$

where the subscript " $G$ " denotes the gas phase. Solutions to Eqs. (20) - (23) can be developed in the same fashion as was done for the liquid-phase equations. The solutions for the amplitudes of the $n^{\text {th }}$ mode of each perturbatlon variable are listed below, where the condition that perturbations should vanish as $x \rightarrow \infty$ was applied and also where terms of order $\phi / R$ or smaller were neglected.

$V_{O x}=b_{1} x^{-n}+b_{2} x^{-n-2}$

$T_{O x}=b_{3} x^{-n-1}+O(\phi / R)$

$Y_{18 x}=b_{41} x^{-n-1}+O(\phi / R)$

The amplitude in Eq. (24) is for the radial velocity component. In addition, $b_{1}, b_{2}, b_{3}$ and $b_{41}$ are integration constants.

\section{INTERFACE CONDITIONS}

The solutions for the perturbation quantitles contain nine unknown constants, which can be determined by imposing appropriate jump conditions at the liquild-gas interface. Written in dimensionless form, equations describing the interface conditions in terms of the perturbation amplitudes are listed below.

$V_{L x}=V_{O x}$

$\frac{d}{d x}\left(x^{2} V_{L x}\right)=\frac{\rho_{L}}{\rho_{O}} \frac{d}{d x}\left(x^{2} V_{O x}\right)$

$$
\begin{aligned}
& {\left[\frac{d^{2}}{d x^{2}}+2 \frac{d}{d x}-2+n(n+1)\right] V_{L x}=} \\
& \gamma\left[\frac{d^{2}}{d x^{2}}+2 \frac{d}{d x}-2+n(n+1)\right] V_{L x} \\
& +M_{T} n(n+1) T_{L x}+M_{Y} n(n+1) y_{1 L x} \\
& T_{L X}=T_{G x}
\end{aligned}
$$




$$
\begin{aligned}
& \frac{d T_{O x}}{d x}=\frac{\lambda_{L}}{\lambda_{0}} \frac{d T_{L x}}{d x}+\frac{\phi}{R} \frac{L}{c_{p} T_{\infty}} V_{O x} \\
& \frac{\phi}{R}\left(y_{1 L x}-\varepsilon \frac{d y_{1 L x}}{d x}+y_{1} \cdot V_{L x}\right)= \\
& \frac{\phi}{R}\left(Y_{10 x}+Y_{100} V_{O x}\right)-\frac{d Y_{10 x}}{d x} \\
& \frac{\phi}{R}\left[y_{2 L x}-e \frac{d y_{2 L x}}{d x}+\left(1-y_{1-}\right) V_{L x}\right]= \\
& \frac{\phi}{R}\left(Y_{2 O x}+Y_{200} V_{O x}\right)-\frac{d Y_{2 O x}}{d x} \\
& y_{1 L x}=\Gamma_{1} Y_{10 x}-\frac{y_{1-} L_{1} T_{\infty}}{R_{1} T_{0+}{ }^{2}} T_{L} \\
& y_{2 L x}=\Gamma_{2} X_{20 x}-\frac{\left(1-y_{1-}\right) L_{2} T_{\infty} T_{L}}{R_{2} T_{0+}{ }^{2}}
\end{aligned}
$$

Equation (27) describes overall mass conservation across the interface, while Eq. (28) equates tangential velocities. Equation (29) describes the balance between the gas and liquid shear stresses and the surface tension gradients that occur from temperalure and spocies gradients where $M_{T}=$ $8 R \sigma_{T} d\left(T_{\infty} k \mu_{L}\right)$ and $M_{Y}=8 R \sigma_{Y_{1}} /\left(k \mu_{L}\right)$ are Marangoni numbers and $\gamma=\left(\mu_{0} / \mu_{L}\right)\left(\rho_{L} / \rho_{0}\right)$. Equations (30) equates gas and liquid temperatures, and $\mathrm{Eg}$. (31) is an onergy balance across the interface. Equations (32) and (33) are the interface species conservation equations, while Eqs. (34) and (35) are the linearized versions of the equilibrium condition (Eq. (7)) where $\Gamma_{1}=$ $\exp \left[\mathrm{L}_{1}\left(1 / \mathrm{T}_{0_{+}}-1 / \mathrm{T}_{b_{1}}\right) / \mathrm{R}_{1}\right] \mathrm{W}_{\mathrm{G}} / \mathrm{W}_{\mathrm{L}}$ and $\Gamma_{2}=$ $\exp \left[L_{2}\left(1 / T_{0_{+}}-1 / T_{b 2}\right) / R_{2}\right] W_{o} / W_{L}$ where $L_{1}$ and $L_{2}, R_{1}$ and $R_{21}$ and $T_{b 1}$ and $T_{b 2}$ are the enthalpies of vaporization, gas constants and bolling temperatures of species 1 and 2 , respectively, and $W_{O}$ and $W_{L}$ are average molecular weights for the gas and liquid phases. In deriving Egs. (34) and (35), perturbations in gas and liquid average molecular weights were neglected.
Substituting the gas and liguid solutions into the Interface relations produces nine oquations relating the nino unknown constants. The equations can be expressed in matrix form as $[A][B]=[0]$, where $[B]=$ $\left[a_{1}, a_{2}, a_{3} a_{11}, b_{1}, b_{2}, b_{3}, b_{41}, b_{42}\right]$ is a column vector and $[A]$ is shown on the last page of thls paper. In this matrix, $\Omega_{1}=(n$ 1)/n+MY $\left(y_{1-}-y_{10}\right) /\left(2 \beta_{n}\right), \Omega_{2}=(n+2) /(n+$ 1) $+M_{Y}\left(y_{1}-y_{10}\right) /\left(2 \beta_{n}\right), \Omega_{3}=1+$ $[16 \varepsilon L \theta(2 n+3)]^{-1}, \Omega_{4}=\left(\lambda_{V} / \lambda_{O}\right)(n=(n+$ $2) /[16 \varepsilon \operatorname{Lo}(2 n+3)]], \psi_{1}=y_{1} \cdot L_{1} T_{+\infty}\left(R_{1} T_{0+}{ }^{2}\right)$ and $\psi_{2}=\left(1-y_{1-}\right) L_{2} T_{-} /\left(R_{2} T_{0+}{ }^{2}\right)$.

\section{STABILITY PREDICTIONSIDISCUSSION}

The stability of two-component droplet gaslfication can bo evaluated by selting the determinant of $[A]$ equal to zero and solving for the growth factor $\beta_{n}$. Because of the large number of terms in the matrix, this was accomplished using a symbolic calculation software package [22]. The variable $\beta_{n}$ was found to depend upon a large number of terms (roughly 100), and for brevity the dispersion relation is not listed here.

Surprisingly, it was found that the disporsion relation did not depend upon either of the Marangoni numbers defined earlier $\left(\mathrm{M}_{\mathrm{A}}\right.$ and $M_{Y}$ ); they cancolled out of all calculations in the determinant. This was verified directly by calculating the minors associated with the terms $\Omega_{1}, \Omega_{2},-M_{A} \Omega_{3} / 2$ and $-M_{Y} / 2$ in $[A]$; the minors for $\cdot M_{A} \Omega_{3} / 2$ and $\cdot M_{Y} / 2$ were identically zero, while the minors for $\Omega_{1}$ and $\Omega_{2}$ were exactly equal. In addition, the dispersion relation predicted that droplets should be absolutcly stable, that is, $\beta_{n}<0$ in general. In rotrospect, this result might have been expected. To clarify why this is so, we should recall that the leading-order solution $\left(y_{1}=y_{10}+\left(y_{1}-y_{10}\right) \exp (-z)\right)$ was used for the unperturbed state, while Eq. (3) shows that the leading-order expression for the mass- 
flux fraction of species $i$ leaving the droplet is simply $\delta_{i}=y_{10}$, i.e., $\delta_{1}$ is dependent only upon the initial mass fraction of species $i$ and is independent of variations in other variables. As a result, in this diffusioncontrolled limit for liquid species transport, small perturbations in liquid-phase velocity and species profiles do not influence the mass-flux fraction of a species leaving a droplet, and $\delta_{1}$ will be unchanged over the surface of a droplet as will the liquid-phase composition at the surface. By Eq. (5), the gas-phase solution will therefore be unchanged, and small perturbations in the liquid phase will not significantly alter gasphase specles or temperature fields so long as $\delta_{1}=y_{10}$ is an adequate approximation. Under these conditions, all perturbations die out from viscous decay of velocity fields.

Il should be noted, however, that these conclusions are not expected to be valid for all cases. For example, it is possible for the $O(\varepsilon)$ correction in Eq. (3) to be important, and when this is so the analysis presented here may not be valid. This can happen, for example, when species 1 is initially present in very small amounts and is of low volatillty relative to the other liquld species so that it must build to appreciable levels at the droplet surface before it can vaporize. Another possibllity is when species i was not even present initially in the droplet, but was first absorbed and then gasified from the droplet surface (e.g., water condensation onto alcohol droplets). For analysis of the stability characteristics of droplets under these conditions, further analysis is necessary. These analyses are being performed, and will be publishod at a later date.

Even though these results were derived for slow gasification rates $(\phi / R \ll 1)$, it is expected that they will be valid for rapid gasification $(\phi / R \geq 1)$ as well as for droplet combustion. So long as the liguid species transport is diffusion controlled and $\delta_{\mathrm{i}}=\mathrm{y}_{10}$ is a good approximation for the liquid phase, small perturbations in liquid-phase variables (c.g., velocities) will not significantly affect $\delta_{1}$. As a result, small perturbations in liquidphase. variables will not inducc surface temperature or species gradients, and velocity perturbations will decay from viscous effects.

\section{ACKNOWLEDOEMENT}

Thls research was supported by NASA Grant NCC3-245. Technical supervision was provided by Dr. Vedha Nayagam.

\section{REFERENCES}

1. Landis, R.B., and Mills, A.F., Firth International Heat Transfer Conference, Paper B7.9, Tokyo, Japan, 1974.

2. Law, C.K., A.I.Ch.E.J. 24: 626 (1978).

3. Wang, C.H., Liu, X.Q.. and Law, C.K., Combust. Flame 56: 175 (1984).

4. Randolph, A.L., Makino, A., and Law, C.K., Twenty-First Symp. (Int'l.) on Combustion, The Combustion Institute, Pittsburgh, 601 (1986).

5. Law, C.K., Combust. Sci. Tech. 17: 29 (1977).

6. Law, C.K., Lee, C.H., and Srinivasan, N., Combust. Flame 37: 125 (1980).

7. Niioka, T., and Salo, J., Twenty-First Symp. (Int'l.) on Combustion. The Combustion Institute, Pittsburgh, 625 (1986).

8. Yang, J. C., Jackson, G. S., and Avedisian, C. T., Twenty-Third Symp. (Int'l.) on Combustion, The Combustion Institute, 1619 (1991).

9. Mikami, M., Kono, M., Salo, J., Dietrich, D., L., and Williams, F. A., Combust, Sci. Tech. 90: 111 (1993). 
10. Yang, J. C., and Avedisian, C. T. Twenty-Second Symp. (Int'l.) on Combustion, The Combustion Institute, 2037 (1989).

11. Law, C.K., and Law, H.K., AIAA J. 20: 522 (1982).

12. Law, C.K., and Binark, M., Int. J. Heat Mass Transfer 22: 1009 (1979).

13. Law, C.K., Xleng, T.Y., and Wang, C.H., Int. J. Heat Mass Transfer 30: 1435 (1987).

14. Choi, M.Y., Dryer, F.L., and Haggard, J.B., Jr., Some Further Observations on Micro-gravity Droplet Combustion in the NASA-Lewis Drop Tower Pacilities, Third Intomational Colloquium on Drops and Bubbles, Monteroy, CA, Sept. 1821,1988 .

15. Shaw, B.D. Combust. Flame, 81: pp. 277 (1990).

16. Higueta, F. J. and Linán, A.. Stability of a Droplet Vaporizing in a Hot Atmosphere. Prog. Astro. Aero. 105: 217 (1985).

17. Chai, A.-T., Rashidnia, N., and Arpaci, V. S.(1992). Marangoni Instability Induced Convection in an Evaporating Liquid Droplet. Proceodings VIII European Symposium on Materials and Fluid Sclences in Microgravlty, p. 187. Brussels, Belgium.

18. Lozinski, D., and Matalon, M. Phys. Fluids A 5 (7): 1596-1601 (1994).

19. Niazmand, H., Shaw, B. D., Dwyer, H. A., and Aharon, I., Combust. Scl. Tech. (in press).

20. Shih, A. T., and Megaridis, C. M. (1994). Thermocapillary Motion Erfects on Convective Droplet Evaporation. Paper presented at the 1994 Spring Meeting of the Central States Section of the Combustion Institute, Madison. Wisconsin.
21. Shaw, B.D., and Williams, F.A. Int. J. Heat Mass Trans., 33: 301 (1990).

22. Mathematica. Vcrsion 1.2.2 33 Enhanced. Wolfram Research, Inc. 


\begin{tabular}{|c|c|c|c|c|c|c|c|c|}
\hline 1 & 1 & 0 & 0 & -1 & -1 & 0 & 0 & 0 \\
\hline$\frac{\rho_{Q}}{\rho_{L}}(n+1)$ & $\frac{P_{O}}{P_{L}}(n+3)$ & 0 & 0 & $n-2$ & $n$ & $\therefore 0$ & 0 & 0 \\
\hline $\mathbf{\Omega}_{\mathbf{l}}$ & $\Omega_{2}$ & $\frac{M_{T}}{2} \Omega_{3}$ & $-\frac{M_{Y}}{2}$ & $-\gamma \frac{n-1}{n}$ & $-\gamma \frac{n+2}{n+1}$ & 0 & .0 & 0 \\
\hline 0 & 0 & $\Omega_{3}$ & 0 & 0 & 0 & $-1=$ & 0 & 0 \\
\hline 0 & 0 & $\boldsymbol{\Omega}_{\mathbf{4}}$ & 0 & $\frac{\phi}{R} \frac{L}{c_{p} T_{\infty}}$ & $\frac{\phi}{R} \frac{L}{c_{p} T_{\infty}}$ & $n+1$ & 0 & 0 \\
\hline$\frac{\phi}{\mathrm{R}} \mathrm{y}_{1}$. & $\frac{\phi}{\mathrm{R}} \mathrm{y}_{1}$. & 0 & $\frac{\phi}{R}\left(1+s_{4}\right)$ & $-\frac{\phi}{R} Y_{1 O_{0}}$ & $-\frac{\phi}{R} Y_{10_{0}}$ & 0 & $-n \cdot 1 \cdot \frac{\phi}{R}$ & 0 \\
\hline$\frac{\phi}{R}\left(1-y_{1}\right)$ & $\frac{\phi}{R}\left(1-y_{1-}\right)$ & 0 & $-\frac{\phi}{R}(1+\$ 4)$ & $-\frac{\phi}{R} Y_{20}$ & $-\frac{\phi}{R} Y_{2 a_{0}}$ & 0 & 0 & $-n-1-\frac{\phi}{R}$ \\
\hline$\frac{y_{1}-y_{10}}{\beta_{n}}$ & $\frac{Y_{1} \cdot y_{10}}{\beta_{n}}$ & $-\psi_{1} \Omega_{3}$ & -1 & 0 & 0 & 0 & $\Gamma_{1}$ & 0 \\
\hline$\frac{y_{1 \cdot} \cdot y_{10}}{\beta_{n}}$ & $\frac{y_{1} \cdot y_{10}}{\beta_{n}}$ & $\psi_{2} \Omega_{3}$ & -1 & 0 & 0 & 0 & 0 & $\Gamma_{2}$ \\
\hline
\end{tabular}

The Matrix [A] 
TECHNICAL PUBLICATION REVIEW AND APPROVAL RECORD AND PROCESSING INFORMATION

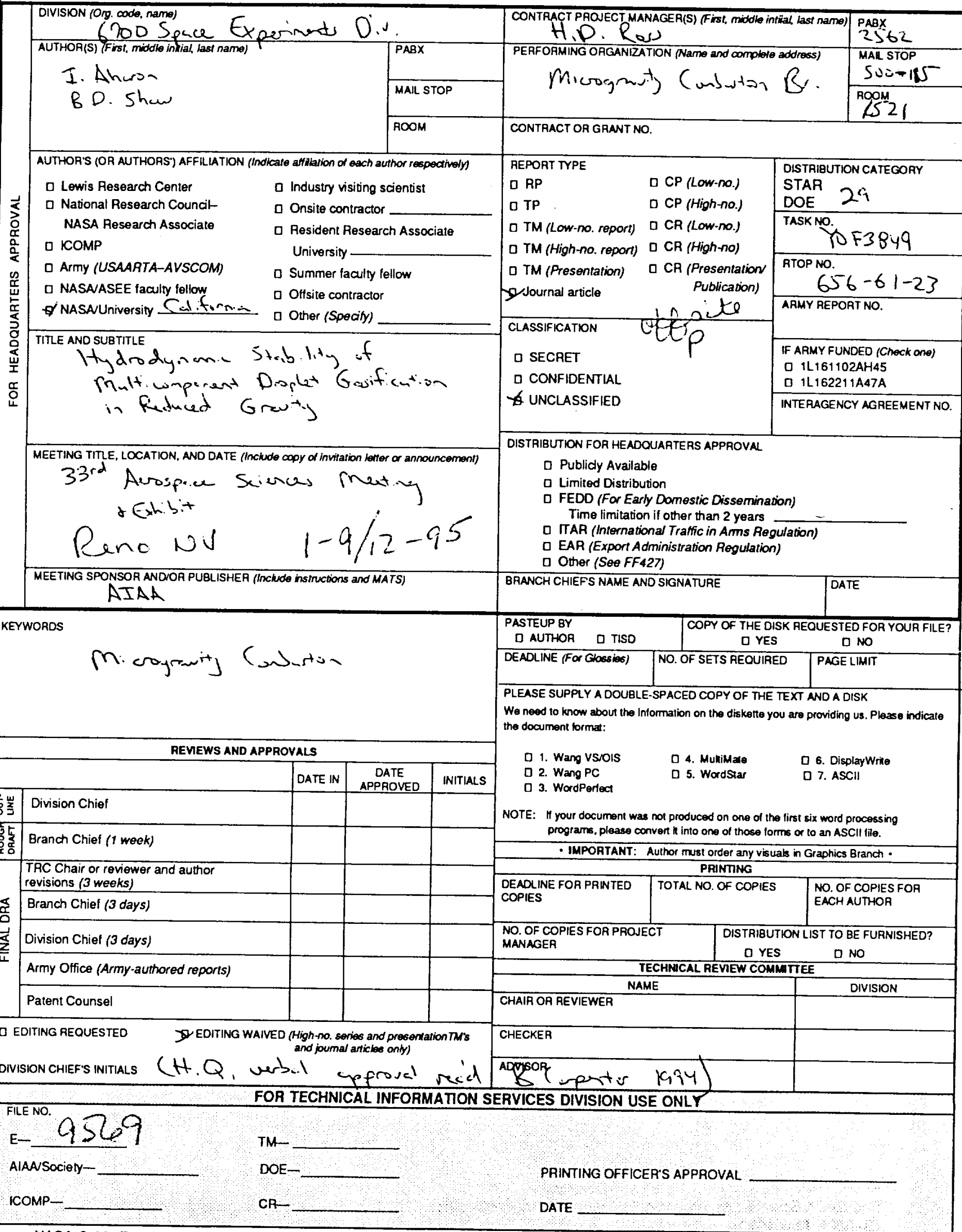

NASA-C-22 (Rev. 6-90) Previous editions are obsolete.

COPY DISTRIBUTION: WHITE-Author's Division
Lewis Stock No. 7530-00-LNO-0218

CARBON-Report Control 